\title{
Involvement of Rho-kinase in TGF- $\beta$-stimulated heat shock protein 27 induction in osteoblasts
}

\author{
HIDEO NATSUME $^{1,2}$, JUN MIZUTANI ${ }^{1}$, SEIJI ADACHI ${ }^{2}$, RIE MATSUSHIMA-NISHIWAKI ${ }^{2}$, \\ CHIHO MINAMITANI ${ }^{1,2}$, HARUHIKO TOKUDA $^{2,3}$, OSAMU KOZAWA ${ }^{2}$ and TAKANOBU OTSUKA ${ }^{1}$ \\ ${ }^{1}$ Department of Orthopedic Surgery, Nagoya City University Graduate School of Medical Sciences, \\ Nagoya 467-8601; ${ }^{2}$ Department of Pharmacology, Gifu University Graduate School of Medicine, \\ Gifu 501-1194; ${ }^{3}$ Department of Clinical Laboratory, National Hospital for Geriatric Medicine, \\ National Center for Geriatrics and Gerontology, Obu 474-8511, Japan
}

Received March 13, 2009; Accepted June 29, 2009

DOI: $10.3892 / \mathrm{mmr} 00000157$

\begin{abstract}
We previously reported that transforming growth factor- $\beta$ (TGF- $\beta$ ) stimulates heat shock protein 27 (HSP27) induction through $\mathrm{p} 44 / \mathrm{p} 42$ mitogen-activated protein (MAP) kinase, p38 MAP kinase and stress-activated protein kinase/ c-Jun N-terminal kinase (SAPK/JNK) in osteoblast-like MC3T3-E1 cells. In addition, we recently reported that the Rho-kinase inhibitors Y27632 and fasudil suppressed the TGF- $\beta$-induced phosphorylation of SAPK/JNK, but not $\mathrm{p} 44 /$ p42 MAP kinase, p38 MAP kinase or Smad2. In the present study, to investigate whether Rho-kinase is involved in TGF- $\beta$ stimulated HSP27 induction in MC3T3-E1 cells, we examined the effects of Rho-kinase inhibitors on HSP27 induction. Y27632 and fasudil significantly suppressed the HSP27 induction stimulated by TGF- $\beta$ in a dose-dependent manner, without affecting the protein levels of HSP70 or HSP90. Immunofluorescence microscopy also revealed that TGF- $\beta$ clearly stimulated, while Y27632 and fasudil markedly suppressed, HSP27 induction in the cytosol of these cells. Taken together, these findings indicate that Rho-kinase regulates TGF- $\beta$-stimulated HSP27 induction via $\mathrm{SAPK} / \mathrm{JNK}$ activation in osteoblasts.
\end{abstract}

\section{Introduction}

It is generally recognized that heat shock proteins (HSPs) are stimulated in cells in response to biological stress, such as heat stress and chemical stress (1). Based on their apparent molecular size, HSPs are classified into high-molecular-weight

Correspondence to: Dr Jun Mizutani, Department of Orthopedic Surgery, Nagoya City University Graduate School of Medical Sciences, Nagoya 467-8601, Japan

E-mail: mjun@med.nagoya-cu.ac.jp

Key words: Rho-associated kinase, transforming growth factor- $\beta$, heat shock protein 27 , osteoblast
HSPs, such as HSP70 and HSP90, and low-molecular-weight HSPs. Low-molecular-weight HSPs with a molecular mass of 10-30 kDa, such as HSP27, $\alpha \mathrm{B}$-crystallin and HSP20, share high homology in amino acid sequences ( $\alpha$-crystallin domain) (2). Though the function of low-molecular-weight HSPs is not as well understood as that of high-molecular-weight HSPs, it is currently believed that the low-molecular-weight HSPs have chaperone functions similar to those of the high-molecularweight HSPs (2). HSP27 activity is reportedly regulated by post-translational modifications, such as phosphorylation (3). Under unstimulated conditions, HSP27 exists in a highmolecular-weight aggregated form and is rapidly dissociated as a result of phosphorylation $(4,5)$. The phosphorylation-elicited dissociation from the aggregated form correlates with loss of molecular chaperone activity $(4,5)$.

Bone metabolism is regulated by two functional cells, osteoblasts and osteoclasts, responsible for bone formation and bone resorption, respectively (6). The maintenance of bone structure and bone remodeling result from the coupling process; bone resorption by activated osteoclasts with subsequent deposition of new matrix by osteoblasts. In osteoblasts, it has been shown that the down-regulation of proliferation is accompanied by a transient increase in HSP27 mRNA expression (7). In addition, heat-stimulated induction of HSP27 is reportedly facilitated by estrogen (8). These findings lead us to speculate that HSP27 participates in the coordination of osteoblast function. However, so far the exact role of HSP27 in osteoblasts remains to be clarified.

It is known that transforming growth factor- $\beta$ (TGF- $\beta$ ) regulates cell growth, differentiation and extracellular matrix production (9). TGF- $\beta$, which is abundantly stored in bone matrix tissue, stimulates the recruitment and proliferation of osteoblasts (10). The intracellular signaling of TGF- $\beta$ is initiated following ligand binding to the TGF- $\beta$ type II receptor, which activates TGF- $\beta$ type I receptor (11). The activated type I receptor phosphorylates Smad2 and 3, causing them to translocate to the nucleus. There, they bind to DNA in the promoters of TGF- $\beta$ target genes (12). In addition to the Smad signaling pathway, other signaling pathways, such as the mitogen-activated protein (MAP) kinase superfamily, have 
recently been shown to mediate TGF- $\beta$ signaling (13). Three major MAP kinases, namely p44/p42 MAP kinase, p38 MAP kinase and stress-activated protein kinase/c-Jun N-terminal kinase (SAPK/JNK), are known to be the central elements used by mammalian cells to transduce diverse messages (14). In previous studies $(15,16)$, we showed that TGF- $\beta$ stimulated HSP27 induction via p38 MAP kinase, p44/p42 MAP kinase and SAPK/JNK in osteoblast-like MC3T3-E1 cells. However, the precise roles of the MAP kinase superfamily in the TGF- $\beta$ signaling system in osteoblasts remain to be clarified.

It is generally recognized that Rho and its downstream effector Rho-associated kinase (Rho-kinase) play important roles in a variety of cellular functions, including cell motility and smooth muscle contraction (17-19). With regard to osteoblasts, it has been demonstrated that Rho and p38 MAP kinase are involved in the endothelin-1-induced expression of prostaglandin endoperoxide $\mathrm{G} / \mathrm{H}$ synthase mRNA in osteoblasts (20). In addition, it has been shown that the Rho/Rho-kinase pathway stimulates osteoblast proliferation while inhibiting osteoblast differentiation (21). In a previous study (22), we reported that Rho-kinase functions as a positive regulator of the endothelin-1-induced synthesis of interleukin-6, a potent bone resorptive agent, in osteoblast-like MC3T3-E1 cells.

In the present study, we investigated the involvement of Rho-kinase in TGF- $\beta$-stimulated HSP27 induction in MC3T3-E1 cells.

\section{Materials and methods}

Materials. TGF- $\beta$ was obtained from R\&D Systems, Inc. (Minneapolis, MN). Y27632 was obtained from CalbiochemNovabiochem Co. (La Jolla, CA). Hydroxyfasudil (fasudil) was purchased from Sigma (St. Louis, MO). Antibodies against HSP27, HSP70 and HSP90 for Western blot analysis were obtained from R\&D Systems. Anti-glyceraldehyde 3 phosphate dehydrogenase (GAPDH) antibodies for Western blot analysis and anti-HSP27 antibodies for immunofluorescence microscopy were obtained from Santa Cruz Biotechnology, Inc. (Santa Cruz, CA). Alexa Fluor $488^{\circledR}$-conjugated calf antigoat antibodies and Alexa Fluor $555^{\circledR}$ phalloidin were obtained from Invitrogen Corp., Inc. (Carlsbad, CA). DAPI was obtained from Wako Pure Chemical Industries, Ltd. (Tokyo, Japan). The ECL Western Blotting Detection System was purchased from GE Healthcare UK Ltd. (Buckinghamshire, UK). Other materials and chemicals were obtained from commercial sources. Y27632 was dissolved in dimethyl sulfoxide. The maximum concentration of dimethyl sulfoxide was $0.1 \%$, which did not affect the assay for Western blot analysis or immunofluorescence microscopy.

Cell culture. Cloned osteoblast-like MC3T3-E1 cells derived from newborn mouse calvaria were maintained as previously described (23). Briefly, the cells were cultured in $\alpha$-minimum essential medium ( $\alpha$-MEM) containing $10 \%$ fetal calf serum (FCS) at $37^{\circ} \mathrm{C}$ in a humidified atmosphere of $5 \% \mathrm{CO}_{2} / 95 \%$ air. The cells were seeded into $90-\mathrm{mm}$ dishes $\left(20 \times 10^{4} / \mathrm{dish}\right)$ for Western blot analysis or $35-\mathrm{mm}$ glass-bottom dishes $\left(5 \times 10^{4} /\right.$ dish) for immunofluorescence microscopy. After 5 days, the medium was exchanged with $\alpha$-MEM containing $0.3 \%$ FCS. The cells were used in the experiments after $48 \mathrm{~h}$.
Western blot analysis. Western blot analysis was performed as described previously (24). The cultured cells were pretreated with various doses of Y27632 or fasudil for $60 \mathrm{~min}$, and then stimulated by TGF- $\beta$ in the presence of inhibitors in $\alpha$-MEM containing $0.3 \%$ FCS for the indicated time periods. The cells were washed twice with phosphate-buffered saline and subsequently lysed, homogenized and sonicated in a lysis buffer containing $62.5 \mathrm{mM}$ Tris/ $\mathrm{HCl}$ (pH 6.8), 3\% sodium dodecyl sulfate (SDS), $50 \mathrm{mM}$ dithiothreitol and $10 \%$ glycerol. SDS-polyacrylamide gel electrophoresis (PAGE) was performed according to Laemmli in $10 \%$ polyacrylamide gel $(25)$. The protein $(20 \mu \mathrm{g})$ was fractionated and transferred onto an Immun-Blot PVDF Membrane (Bio-Rad, Hercules, CA). Membranes were blocked with $5 \%$ fat-free dry milk in Tris-buffered saline-Tween-20 [TBS-T; $20 \mathrm{mM}$ Tris/HCl (pH 7.6), $137 \mathrm{mM} \mathrm{NaCl}$ and $0.1 \%$ Tween-20] for $2 \mathrm{~h}$ before incubation with the primary antibodies. Western blot analysis was performed using anti-HSP27, anti-HSP70, anti-HSP90 and anti-GAPDH antibodies with peroxidase-labeled antibodies raised in goat anti-rabbit IgG which were used as secondary antibodies. Peroxidase activity on the PVDF membranes was visualized on X-ray film by means of the ECL Western Blot Detection System and was quantitated using NIH image software. All Western blot analyses were repeated at least three times in independent experiments.

Immunofluorescence microscopy. The cultured cells were pretreated with various doses of Y27632 or fasudil at the indicated concentrations for $1 \mathrm{~h}$, then exposed to TGF- $\beta$ $(10 \mathrm{ng} / \mathrm{ml})$ or vehicle for $12 \mathrm{~h}$. They were then fixed with $3 \%$ paraformaldehyde for $10 \mathrm{~min}$ on ice and exposed to $0.1 \%$ Triton X-100 for $10 \mathrm{~min}$ to permeabilize the cell membrane. Subsequently, the cells were exposed to anti-HSP27 antibodies (1:100 dilution) in the presence of $1 \%$ BSA for $1 \mathrm{~h}$, followed by exposure to Alexa Fluor 488-conjugated calf anti-goat IgG antibodies (1:500) for $1 \mathrm{~h}$. Finally, they were exposed to Alexa Fluor 555 phalloidin and DAPI for $20 \mathrm{~min}$. The cells were examined using a fluorescence microscope (Biorevo BZ-9000; Keyence, Tokyo, Japan) according to the manufacturer's protocol.

Statistical analysis. Quantification analysis of the indicated protein was calculated as follows: the background was first subtracted from the signal intensity (total optical density) of each protein signal, and each value was normalized to GAPDH or total protein and expressed as relative signal intensity with respect to the control. All data are presented as the mean \pm SEM of triplicate determinations. Data were analyzed by ANOVA followed by the Bonferroni method for multiple comparisons between pairs, and a p-value $<0.05$ was considered significant.

\section{Results}

Effects of Y27632 and fasudil on HSP27 induction stimulated by TGF- $\beta$ in MC3T3-E1 cells. We previously reported that TGF- $\beta$ stimulated HSP27 induction in osteoblast-like MC3T3-E1 cells (15). In the present study, we examined the effect of Y27632, a specific inhibitor of Rho-kinase (19), on HSP27 induction stimulated by TGF- $\beta$. The cells were pretreated with Y27632 and then exposed to TGF- $\beta$, then the expression levels of 

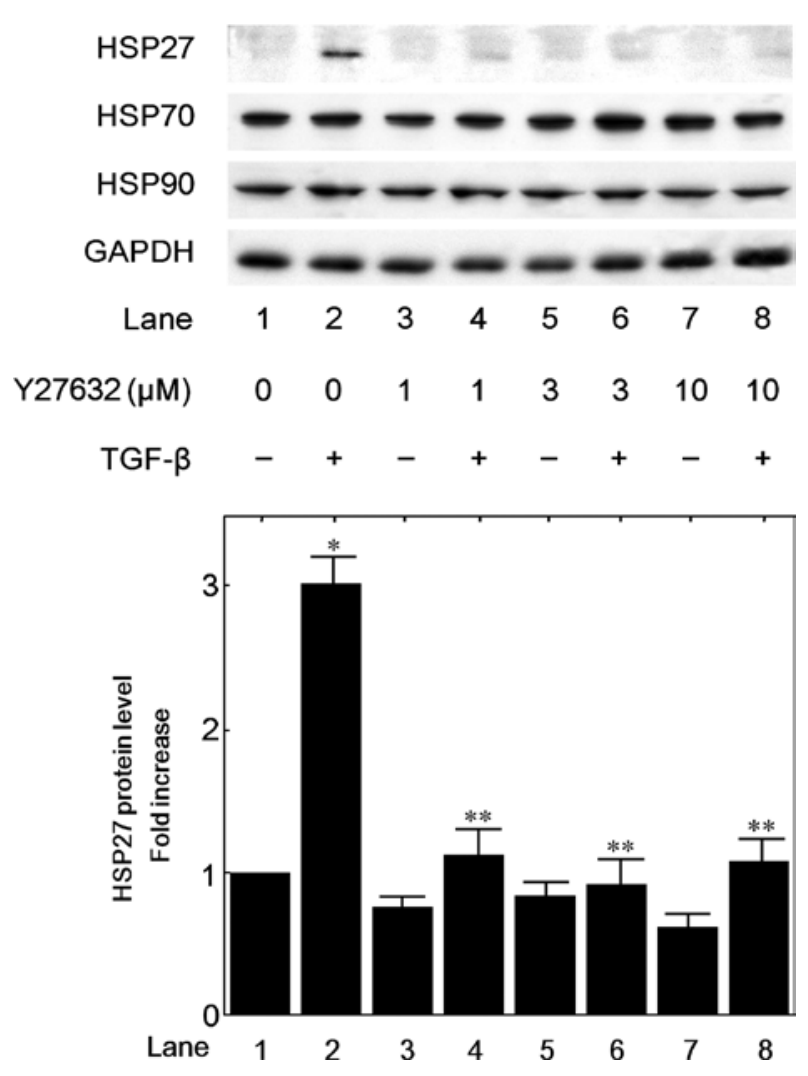

Figure 1. Effect of Y27632 on TGF- $\beta$-stimulated HSP27 induction in MC3T3-E1 cells. The cultured cells were pretreated with various doses of Y27632 for $60 \mathrm{~min}$ and subsequently stimulated by $10 \mathrm{ng} / \mathrm{ml} \mathrm{TGF}-\beta$ or vehicle for $12 \mathrm{~h}$. The extracts of cells were subjected to SDS-PAGE with subsequent Western blot analysis with antibodies against HSP27, HSP70, HSP90 and GAPDH. The lower histogram shows quantitative representations of the levels of TGF- $\beta$-induced HSP27. "p $<0.05$ compared with the control. * $p<0.05$ compared with the value of TGF- $\beta$ alone.

HSP27, 70 and 90 were measured using Western blot analysis (Fig. 1). Y27632 significantly suppressed TGF- $\beta$-stimulated HSP27 induction in a dose-dependent manner in the range of 1-10 $\mu \mathrm{M}$ (Fig. 1, upper panel). We also found that fasudil dose dependently reduced TGF- $\beta$-stimulated HSP27 induction (Fig.2, upper panel). We previously showed that TGF- $\beta$ does not affect the level of HSP70, a high-molecular-weight HSP, in MC3T3-E1 cells (15). In the present study, TGF- $\beta$ had little effect on HSP70 and HSP90 levels (Figs. 1 and 2, lane 1 in comparison with lane 2, respectively). In addition, we found that protein levels of HSP70 and HSP90 were not influenced by treatment with either Y27632 or fasudil alone (Figs. 1 and 2).

Effect of Y27632 and fasudil on TGF- $\beta$-stimulated HSP27 induction in MC3T3-E1 cells. We examined the effect of Y27632 and fasudil on HSP27 induction stimulated by TGF- $\beta$ using immunofluorescence microscopy. As shown in Fig. 3, we found that TGF- $\beta$ clearly stimulated HSP27 induction (green signal) in the cytosol of these cells (Fig. 3; panel 5 in comparison with panel 1), consistent with the results shown in Figs. 1 and 2. Three micromoles of either Y27632 or fasudil, which by itself had no effect on HSP27 induction (data not shown), clearly suppressed HSP27 induction stimulated by TGF- $\beta$ (Fig. 3; panels 9 and 13 in comparison with panel 5). This is also consistent with the results shown in Figs. 1 and
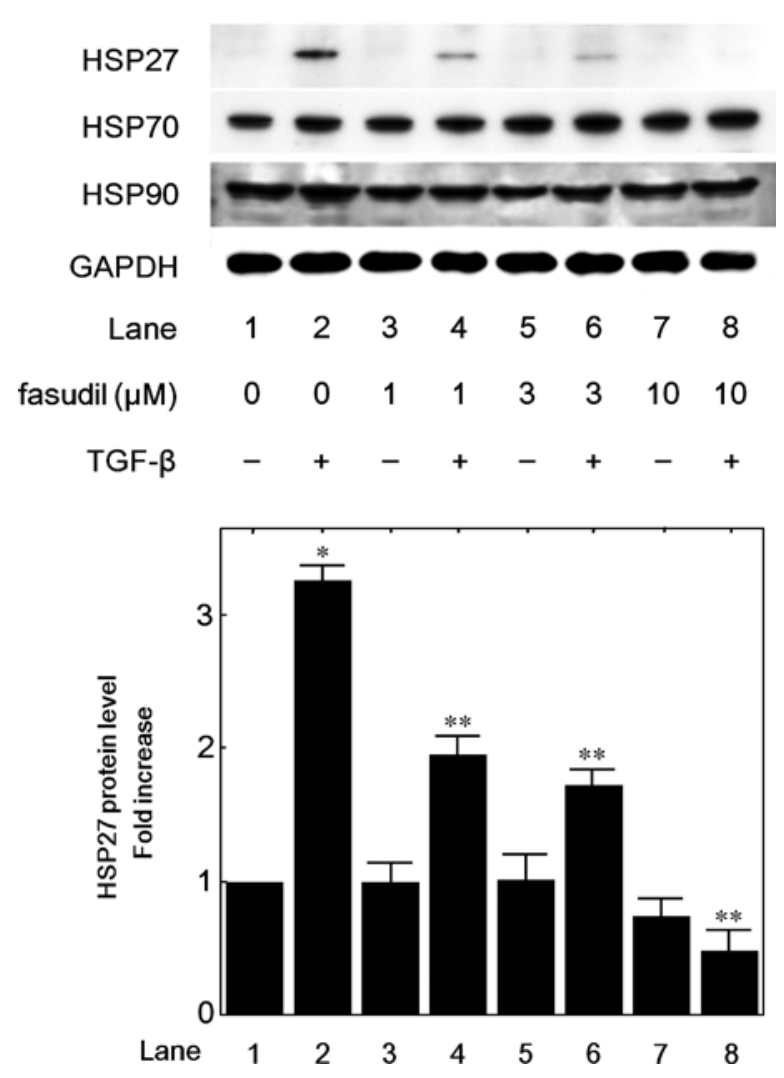

Figure 2. Effect of fasudil on TGF- $\beta$-stimulated HSP27 induction in MC3T3-E1 cells. The cultured cells were pretreated with various doses of fasudil for $60 \mathrm{~min}$ and subsequently stimulated by $10 \mathrm{ng} / \mathrm{ml}$ TGF- $\beta$ or vehicle for $12 \mathrm{~h}$. The extracts of cells were subjected to SDS-PAGE with subsequent Western blot analysis with antibodies against HSP27, HSP70, HSP90 and GAPDH. The lower histogram shows quantitative representations of the levels of TGF- $\beta$-induced HSP27. " $\mathrm{p}<0.05$ compared with the control. ${ }^{* *} \mathrm{p}<0.05$ compared with the value of TGF- $\beta$ alone.

2. In addition, since HSP27 is known to be associated with the cytoskeleton, we examined the effects of Y27632 and of fasudil on actin polymerization using phalloidin, which reveals F-actin (red signal) (26). However, no significant differences in actin polymerization were found (Fig. 3; panels 2, 6, 10 and 14). Moreover, there were no significant differences between the appearance of Y27632- or fasudil-treated cells and the control cells throughout the experiments.

\section{Discussion}

In the present study, we first demonstrated that Y27632 and fasudil significantly suppressed the TGF- $\beta$-stimulated induction of HSP27, a low-molecular-weight HSP, in osteoblast-like MC3T3-E1 cells (Figs. 1 and 2). Moreover, using immunofluorescence microscopy, we confirmed that Y27632 and fasudil markedly reduced TGF- $\beta$-stimulated HSP27 induction (Fig. 3). In our previous study (15), HSP70 was found to be highly expressed in the absence of TGF- $\beta$ in MC3T3-E1 cells. In the present study, we demonstrated that HSP90 was also highly expressed in the absence of TGF- $\beta$ in these cells. Taken together, these results suggest that HSP70 and HSP90 act as housekeeping molecules in osteoblast-like cells.

We previously showed that Rho-kinase functions at a point upstream of SAPK/JNK in TGF- $\beta$-induced VEGF synthesis in 


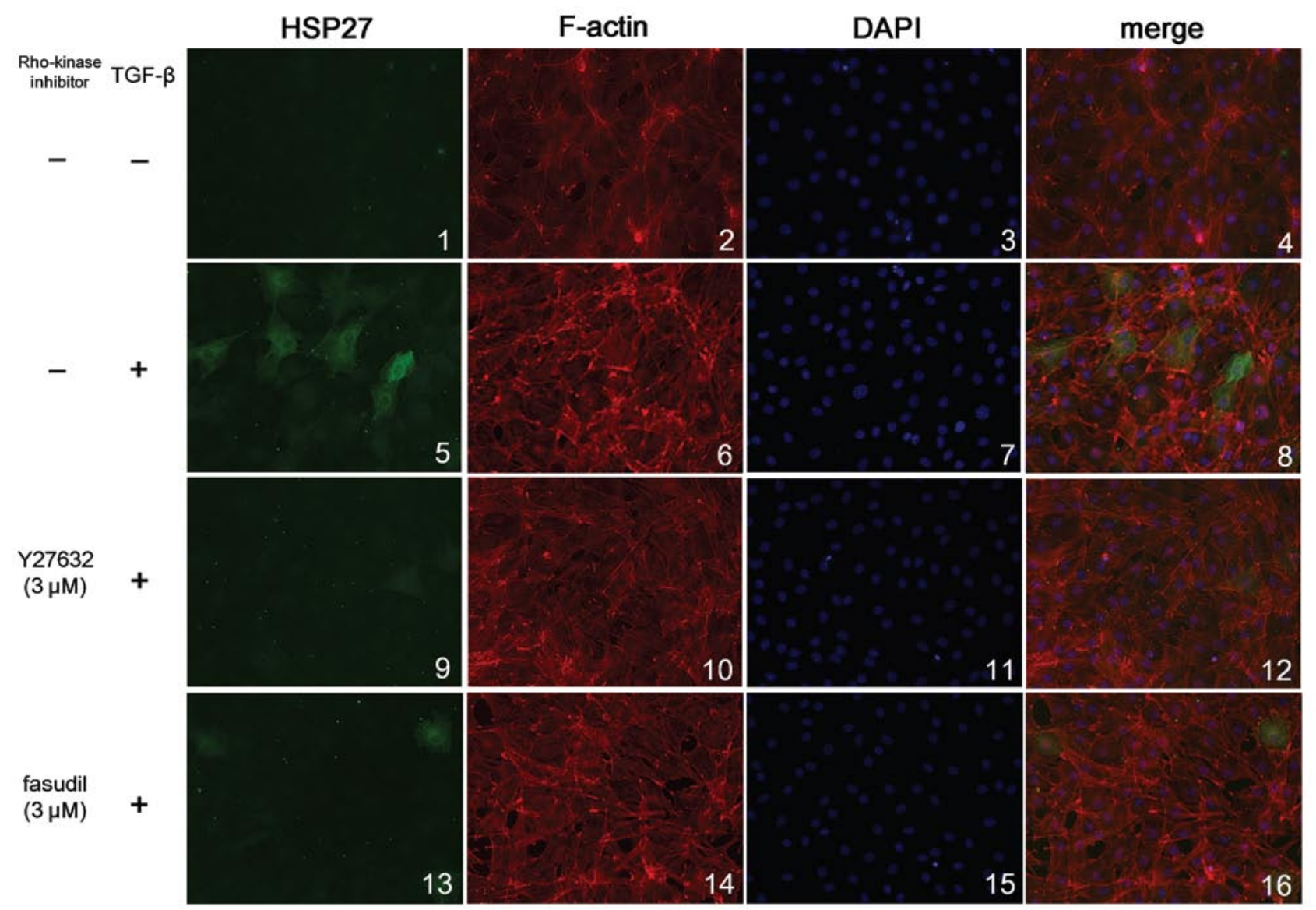

Figure 3. Immunofluorescence microscopy of the expression of HSP27. Cultured cells were pretreated with $3 \mu \mathrm{M}$ Y27632 (panels 9-12), $3 \mu \mathrm{M}$ fasudil (panels 13-16) or vehicle (panels 1-8) for $60 \mathrm{~min}$, followed by stimulation with $10 \mathrm{ng} / \mathrm{ml}$ of TGF- $\beta$ (panels 5-16) or vehicle (panels $1-4$ ) for 12 h. Cell extracts were fixed with paraformaldehyde. After permeabilization with $0.1 \%$ Triton X-100, the cells were exposed to HSP27 antibodies (1:100 dilution) and then treated with Alexa Fluor 488-conjugated anti-goat secondary antibodies (green signal). Lastly, cells were exposed to Alexa Fluor 555 phalloidin for F-actin (red signal) and DAPI for the nucleus (blue signal) for $20 \mathrm{~min}$, then examined by confocal microscopy.

osteoblast-like MC3T3-E1 cells (27). Therefore, it is probable that the inhibition of TGF- $\beta$-stimulated HSP27 induction by Y27632 or fasudil is mediated through the suppression of the SAPK/JNK pathway in MC3T3-E1 cells. In addition, we reported that neither Y27632 nor fasudil affects the activation of p44/p42 MAP kinase or p38 MAP kinase stimulated by TGF- $\beta$ in these cells (27). Therefore, it seems unlikely that Rho-kinase affects TGF- $\beta$-stimulated HSP 27 induction through the modulation of p44/p42 MAP kinase or p38 MAP kinase. Collectively, our results strongly suggest that, in osteoblast-like MC3T3-E1 cells, Rho-kinase acts at a point upstream of SAPK/JNK in the MAP kinase superfamily in TGF- $\beta$-stimulated HSP27 induction.

It is currently recognized that Rho-kinase plays an important role in a variety of cellular functions, particularly vascular smooth muscle contraction (17-19). In bone metabolism, the activation of Rho-kinase reportedly suppresses the differentiation of osteoblasts and induces their proliferation (21). It is well known that TGF- $\beta$ is synthesized in osteoblasts, stored abundantly in bone matrix in its latent form and activated in the bone microenvironment (10). During bone resorption, TGF- $\beta$ is released and stimulates the recruitment and proliferation of osteoblasts. Our present results show that Rho-kinase stimulated by TGF- $\beta$ acts as a positive regulator in HSP27 induction in osteoblasts. It is generally recognized that the main function of most HSPs, including HSP27, is their chaperone function. This includes protein folding, oligomerization and translocation (2). In addition, intensive deposition of extracellular matrix is observed during bone remodeling. Moreover, it has been reported that, in osteoblastlike MC3T3-E1 cells, TGF- $\beta$ causes an increase in the steady state level of HSP47 mRNA, which is known to be a procollagen/collagen-specific molecular chaperone protein (28). These findings lead us to speculate that TGF- $\beta$-induced HSP27 induction assists in the folding of newly synthesized bone matrix proteins by TGF- $\beta$. Although the physiological significance of HSP27 in osteoblasts has not yet been clarified, our finding that SAPK/JNK, but not p44/p42 MAP kinase or p38 MAP kinase, is solely regulated by Rho-kinase suggests the importance of fine tuning MAP kinase-mediated HSP27 induction stimulated by TGF- $\beta$ in bone remodeling. However, the exact role of Rho-kinase in osteoblasts remains unknown. Further investigation including another osteoblast population is necessary to elucidate the exact role of Rho-kinase in bone metabolism.

In conclusion, our results strongly suggest that Rho-kinase inhibitors decrease TGF- $\beta$-stimulated HSP27 induction via the suppression of SAPK/JNK in osteoblasts. 


\section{Acknowledgements}

We are very grateful to Yoko Kawamura for her skillful technical assistance. This investigation was supported in part by Grants-in-Aid for Scientific Research (nos. 16590873 and 16591482) from the Ministry of Education, Science, Sports and Culture of Japan, a Research Grant on Proteomics and a Research Grant on Longevity Sciences from the Ministry of Health, Labour and Welfare of Japan, and a research grant from The Foundation for the Growth of Science.

\section{References}

1. Hendrick JP and Hartl FU: Molecular chaperone functions of heat-shock proteins. Annu Rev Biochem 62: 349-384, 1993.

2. Benjamin IJ and McMillan DR: Stress (heat shock) proteins: molecular chaperones in cardiovascular biology and disease. Circ Res 83: 117-132, 1998.

3. Landry J, Lambert H, Zhou M, Lavoie JN, Hickey E, Weber LA and Anderson CW: Human HSP27 is phosphorylated at serines 78 and 82 by heat shock and mitogen-activated kinases that recognize the same amino acid motif as S6 kinase II. J Biol Chem 267: 794-803, 1992.

4. Kato K, Hasegawa K, Goto S and Inaguma Y: Dissociation as a result of phosphorylation of an aggregated form of the small stress protein, hsp27. J Biol Chem 269: 11274-11278, 1994.

5. Rogalla T, Ehrnsperger M, Preville X, Kotlyarov A, Lutsch G, Ducasse C, Paul C, Wieske M, Arrigo AP, Buchner J and Gaestel M: Regulation of Hsp27 oligomerization, chaperone function, and protective activity against oxidative stress tumor necrosis factor $\alpha$ by phosphorylation. J Biol Chem 274: 18947-18956, 1999.

6. Nijweide PJ, Burger EH and Feyen JH: Cells of bone: proliferation, differentiation, and hormonal regulation. Physiol Rev 66 : 855-886, 1986.

7. Shakoori AR, Oberdorf AM, Owen TA, Weber LA, Hickey E, Stein JL, Lian JB and Stein GS: Expression of heat shock genes during differentiation of mammalian osteoblasts and promyelocytic leukemia cells. J Cell Biochem 48: 277-287, 1992.

8. Cooper LF and Uoshima K: Differential estrogenic regulation of small M(r) heat shock protein expression in osteoblasts. J Biol Chem 269: 7869-7873, 1994.

9. Massague J, Blain SW and Lo RS: TGF $\beta$ signaling in growth control, cancer, and heritable disorders. Cell 103: 295-309, 2000.

10. Bonewald LF: Transforming growth factor- $\beta$. In: Principles of Bone Biology. 2nd edition. Bilezikian JP, Raisz LG and Rodan GA (eds). Academic Press, San Diego, pp903-918, 2002.

11. Miyazono K, ten Dijke P and Heldin CH: TGF- $\beta$ signaling by Smad proteins. Adv Immunol 75: 115-157, 2000.

12. Miyazono K, Kusanagi K and Inoue H: Divergence and convergence of TGF- $\beta$ /BMP signaling. J Cell Physiol 187: 265-276, 2001.

13. Moustakas A and Heldin CH: Non-Smad TGF- $\beta$ signals. J Cell Sci 118: 3573-3584, 2005 .
14. Kyriakis JM and Avruch J: Mammalian mitogen-activated protein kinase signal transduction pathways activated by stress and inflammation. Physiol Rev 81: 807-869, 2001.

15. Hatakeyama D, Kozawa O, Niwa M, Matsuno H, Ito H, Kato K, Tatematsu N, Shibata T and Uematsu T: Upregulation by retinoic acid of transforming growth factor- $\beta$-stimulated heat shock protein 27 induction in osteoblasts: involvement of mitogenactivated protein kinases. Biochim Biophys Acta 1589: 15-30, 2002.

16. Hayashi K, Takai S, Matsushima-Nishiwaki R, Hanai Y, Kato K, Tokuda $\mathrm{H}$ and Kozawa O: (-)-Epigallocatechin gallate reduces transforming growth factor $\beta$-stimulated HSP27 induction through the suppression of stress-activated protein kinase/c-Jun N-terminal kinase in osteoblasts. Life Sci 82: 1012-1017, 2008.

17. Fukata Y, Amano M and Kaibuchi K: Rho-Rho-kinase pathway in smooth muscle contraction and cytoskeletal reorganization of non-muscle cells. Trends Pharmacol Sci 22: 32-39, 2001.

18. Riento K and Ridley AJ: Rocks: multifunctional kinases in cell behaviour. Nat Rev Mol Cell Biol 4: 446-456, 2003.

19. Shimokawa H and Rashid M: Development of Rho-kinase inhibitors for cardiovascular medicine. Trends Pharmacol Sci 28 296-302, 2007.

20. Windischhofer W, Zach D, Fauler G, Raspotnig G, Kofeler H and Leis HJ: Involvement of Rho and p38 MAPK in endothelin-1induced expression of PGHS-2 mRNA in osteoblast-like cells. J Bone Miner Res 17: 1774-1784, 2002.

21. Harmey D, Stenbeck G, Nobes CD, Lax AJ and Grigoriadis AE: Regulation of osteoblast differentiation by Pasteurella multocida toxin (PMT): a role for Rho GTPase in bone formation. J Bone Miner Res 19: 661-670, 2004.

22. Tokuda H, Hanai Y, Matsushima-Nishiwaki R, Yamauchi J, Doi T, Harada A, Takai S and Kozawa O: Rho-kinase regulates endothelin-1-stimulated IL-6 synthesis via p38 MAP kinase in osteoblasts. Biochem Biophys Res Commun 362: 799-804, 2007.

23. Kozawa O, Suzuki A, Tokuda $\mathrm{H}$ and Uematsu T: Prostaglandin $\mathrm{F}_{2 \alpha}$ stimulates interleukin-6 synthesis via activation of PKC in osteoblast-like cells. Am J Physiol 272: E208-E211, 1997.

24. Kato K, Ito H, Hasegawa $\mathrm{K}$, Inaguma $\mathrm{Y}$, Kozawa $\mathrm{O}$ and Asano T: Modulation of the stress-induced synthesis of hsp27 and $\alpha \mathrm{B}$-crystallin by cyclic AMP in C6 rat glioma cells. J Neurochem 66: 946-950, 1996.

25. Laemmli UK: Cleavage of structural proteins during the assembly of the head of bacteriophage T4. Nature 227: 680-685, 1970.

26. Zhu Y, O'Neill S, Saklatvala J, Tassi L and Mendelsohn ME: Phosphorylated HSP27 associates with the activation-dependent cytoskeleton in human platelets. Blood 84: 3715-3723, 1994.

27. Kuno M, Takai S, Matsushima-Nishiwaki R, Minamitani C, Mizutani J, Otsuka T, Harada A, Adachi S, Kozawa O and Tokuda H: Rho-kinase inhibitors decrease TGF- $\beta$-stimulated VEGF synthesis through stress-activated protein kinase/c-Jun $\mathrm{N}$-terminal kinase in osteoblasts. Biochem Pharmacol 77: 196-203, 2009.

28. Yamamura I, Hirata $\mathrm{H}$, Hosokawa $\mathrm{N}$ and Nagata $\mathrm{K}$ : Transcriptional activation of the mouse HSP47 gene in mouse osteoblast MC3T3-E1 cells by TGF- $\beta 1$. Biochem Biophys Res Commun 244: 68-74, 1998. 